\title{
Observing Behavioral Effects in Mini-Pigs after Blocking the Low Hydraulic Resistance Channels along the Stomach Meridian
}

\author{
Wei-Bo Zhang ${ }^{1^{*}}$, Wen-Ting Zhou ${ }^{2}$, Guang-Jun Wang ${ }^{1}$, Shu-Yong Jia ${ }^{1}$, Yu-Ying Tian ${ }^{1}$, Xiao-Jing Song ${ }^{1}$ and Hong-Yan Li ${ }^{1}$ \\ ${ }^{1}$ Institute of Acupuncture and Moxibustion, China Academy of Chinese Medical Sciences, PR China \\ 2Institute of Basic Theory for Chinese Medicine, China Academy of Chinese Medical Sciences, PR China
}

"Corresponding author: Wei-Bo Zhang, Institute of Acupuncture and Moxibustion, China Academy of Chinese Medical Sciences, Addr. 16 Nanxiaojie, Dongzhimennei, Beijing 100700, PR China, Tel: 00861064089385; E-mail: 1507307638@qq.com

Received date: October 05, 2017, Accepted date: October 11, 2017, Published date: October 16, 2017

Copyright: ( 2017 Zhang WB. This is an open-access article distributed under the terms of the Creative Commons Attribution License, which permits unrestricted use, distribution, and reproduction in any medium, provided the original author and source are credited.

\begin{abstract}
Objective: To study the relationship between the meridian and diseases by establishing a pathological model and study the effects of blocked channels on the behavior of mini-pigs.

Method: First, we measured the low electrical impedance line along the stomach meridian. Next we measured the hydraulic resistance (HR) around the meridian to find points with the lowest HR. Gel were then injected into the points to establish a model for "blocked meridian". A control group was injected with saline. The behaviors of the mini-pigs were observed. The video was then reviewed and frequency of climbing fence in the dtime (FCFD) and frequency of nighttime activity (FNA) were analyzed.
\end{abstract}

Results: When the mean values in various time periods were compared, there was no significant difference between the two groups in the two markers. However, over time, the model group exhibited an upward trend of FCFD which had a correlation coefficient of 0.868 with the modeling ds, i.e. the ds elapsed after establishing the model which was statistically significant. The control group, was -0.045 , which was not statistically significant. There was a similar tendency for FNA over time that the correlation coefficient between FNA and modeling ds was 0.675 $(P<0.1)$ while it was 0.401 in the control group $(P>0.1)$.

Conclusion: Blocking LHRC along stomach meridian produces behavioral changes that are consistent with the pathological symptoms of stomach meridian. These changes are directly correlated with the modeling ds and gradually become more pronounced with the passage of time.

Keywords: Hydraulic resistance; Behavior; Mini-pigs; Stomach meridian

\section{Introduction}

Traditional Chinese medicine (TCM) is a science that is based upon a holistic understanding of the patterns or natural laws of life and illness. Behavior such as bowel movements, food intake and sleep is the external expression of all of the processes of life and most objectively and authentically reflects that state of the body. Abnormal behavior and other markers can directly reveal strengths and weaknesses in the "Zang-fu" of Chinese medicine well before those morphological organs develop organic disease.

In the theory of traditional Chinese medicine, the meridianchannels are a regulatory system that maintains the balance of YinYang, Qi-Blood and their ascending, descending, entering and exiting movements. If the meridian-channels are not flowing freely, yin-yang, qi-blood will become imbalanced and may cause pathological changes in the Zang-fu organs. Any pathological changes in the Zang-fu will be reflected in their functioning or the behavior related to them well before full-blown disease occurs, thus providing the body with an early-warning system.

One important component of the meridian theory in TCM is the correspondence of meridians to the internal Zang-fu, and it is posited that each of the 12 meridian-channels connects to one of the internal organs. Therefore when there is a pathological change in any of the meridians, it will be reflected in both the functions of the corresponding internal organ and in external signs and behavior. For example, the Divine Pivot states: "The diseases stem from foot Yangming channel are: shivering with cold, yawns and stretches oneself often, black colour of the forehead, detestation of man and fire when becoming ill, afraid of hearing the sound made by wood, palpitation and prefering to stay alone with the doors and windows closed. When the disease is severe, the patient may sing loudly in a high place and runs about with his clothes taken off, and he has abdominal distention and borborygmus" [1]. From this list of signs and symptoms, we can see that "sing loudly in a high place and runs about with his clothes taken off," are descriptions of behavior. Therefore, explicating the relationship between the meridian-channels and specific behaviors is an important area of research aimed at elucidating the pathology of the meridian-channels.

Our previous research showed the existence of low hydraulic resistance channels (LHRC) that carry interstitial fluid along the meridians and we postulate that these are one of the physical substrates of the meridian-channels [2]. Injecting gel into these channels effectively blocked the signals transmitted through them and allows us to create a pathological model of channel stasis in mini-pigs [3]. Further research showed that blocking these pathways with gel lowered 
Citation: Zhang WB, Zhou WT, Wang GJ, Jia SY, Tian YY, et al. (2017) Observing Behavioral Effects in Mini-Pigs after Blocking the Low Hydraulic Resistance Channels along the Stomach Meridian. Altern Integr Med 6: 251. doi:10.4172/2327-5162.1000251

Page 2 of 5

the pain threshold along the meridians after a certain time had elapsed thus corroborating the principle of "when there is blockage there is pain" that is fundamental in Chinese medical theory [4]. Another study showed that blocking LHRC along the stomach meridian in mini-pigs causes pathological changes such as bloating in the stomach and intestines that are classically attributed to pathology of the stomach meridian and stomach-fu. This is preliminary evidence for the relationship between a meridian-channel and its pertaining organ [5]. We therefore designed the following experiment in order to explore whether blocking a particular meridian-channel would produce corresponding behavioral changes.

\section{Materials and Methods}

\section{Materials}

Experimental animals: Chinese experimental mini-pigs provided by the Beijing Kexing Breeding and Housing Center for Experimental Animals were used. The animals were about 3 months of age when taken into the animal laboratory, weighing from $8 \mathrm{~kg}$ to $10 \mathrm{~kg}$, with black fur. They were all quarantined and ascertained to be healthy animals and were randomly assigned to the experimental or the control group.

Feeding and environment of the animals: The dedicated animal laboratory and cages provided by the Animal Experimentation center of the Institute of Chinese Materia Medica, China Academy of Chinese Medical Sciences(license number: SYXK Beijing 2011-0014) were used (Figure 1A). The cages were $1.1 \mathrm{~m} \times 0.7 \mathrm{~m} \times 0.8 \mathrm{~m}$ and completely separated from one another (Figure 1B). Animal feed was provided by Beijing Xiwang Animal Feed Co. Ltd. We used the Xiwang \#1 puffed kernals pig feed from the latter. They were fed $250 \mathrm{~g}$ per $\mathrm{d}$ : $100 \mathrm{~g}$ in the morning and $150 \mathrm{~g}$ in the evening. Water was available in unlimited quantities. The temperature of the laboratory was $26^{\circ} \mathrm{C}$ to $28^{\circ} \mathrm{C}$ and the lights were set to imitate changes in dtime and nighttime light, with lights coming on at 8 am and turning off at $8 \mathrm{pm}$.

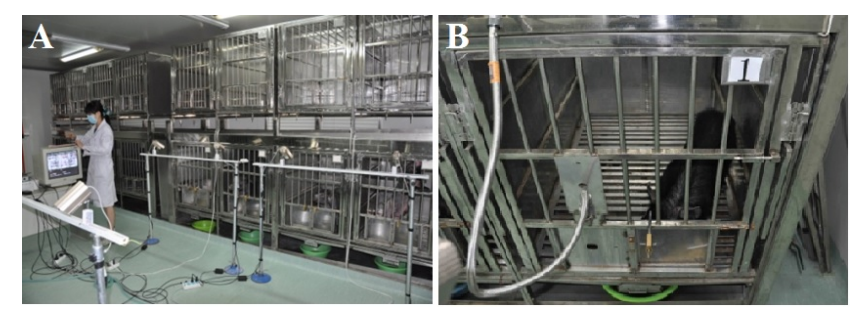

Figure 1: Feeding condition for the experimental mini-pigs. (A) Special room for large experimental animal with camera, monitor and recorder. (B) Special cage for experimental mini-pig with food trough water mouth for free drink.

Surveillance system for pig's behaviors: We used an embedded numerical hard drive video system (model HB7008KC from Beijing Chengyi Wang Ke Information and Technology Company) with 4 cameras for 24 hour continuous surveillance on the 4 mini-pigs in the laboratory (Figure 2). After the lights were turned off, the cameras automatically shifted into infrared mode. All of the behaviors of each pig, including standing, feeding, sleeping, and all others, were captured on video and preserved in the hard drive.

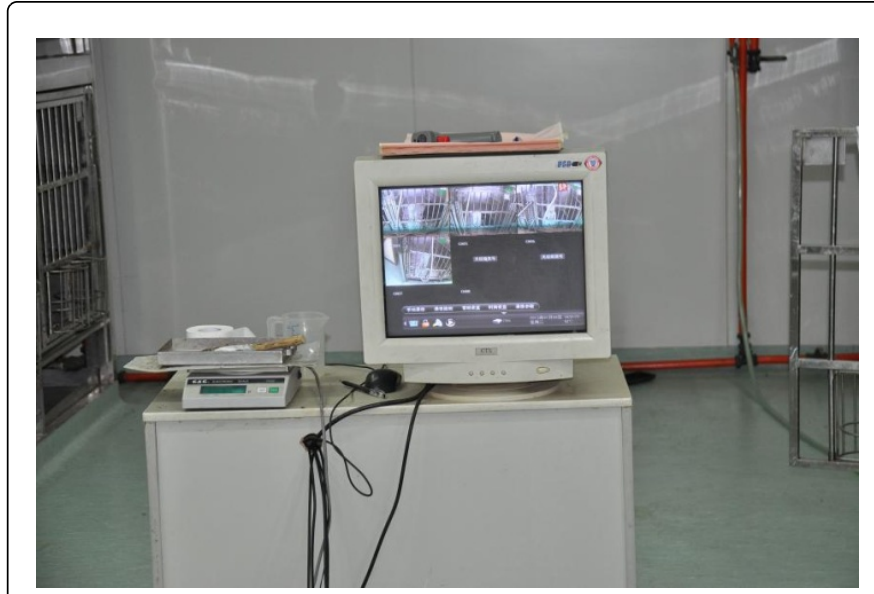

Figure 2: Embedded numerical hard drive video system Model HB7008KC.

Preparation of gel: Stable, translucent gel with the brand name Macrolane (manufactured in Sweden) was used. $5 \mathrm{ml}$ of gel was diluted with $1 \mathrm{ml}$ of physiological saline and a trace amount of Alcian Blue 8 GX (manufactured by SIGMA in the United States) was added to give the gel color. The color enabled us to observe the effects of the gel on blocking the pathways. $0.3 \mathrm{ml}$ of dye was used for every $20 \mathrm{ml}$ of gel.

\section{Methods}

Selection of stomach meridian pathological behavior in mini-pigs: According to our previous experience of behavior study [6], frequency of climbing a small fence in the dtime(FCFD) and frequency of nighttime activity(FNA)were selected as two behavior markers.

Definition of behavioral markers: Lights in the lab were turned off at $8 \mathrm{pm}$ and the mini-pigs were generally soundly asleep by $10 \mathrm{pm}$ (defined as lying on their side or prone for an extended period with deep, steady breathing). The lights were turned on at 8 am, we therefore defined "nighttime" as the interval between $10 \mathrm{pm}$ and $8 \mathrm{am}$.

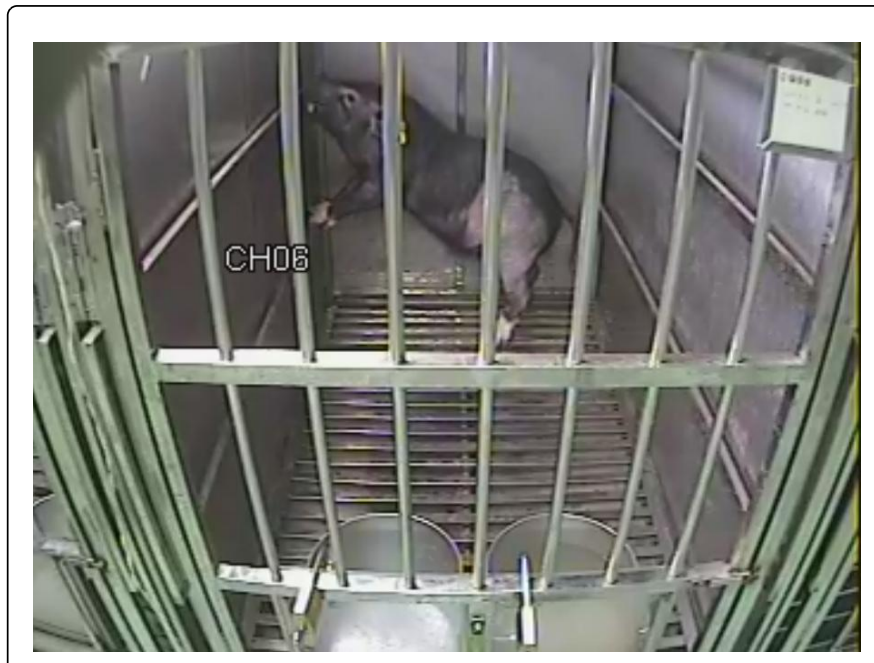

Figure 3: Demonstrate that a mini-pig climbed the fence in the dtime. 


\section{Establishing a model of pathology for "blocked channels"}

Frequency of climbing fence in the dtime (FCFD): defined as any time a mini-pig had two fore hooves off the ground and up the wall (Figure 3). Continuous climbing was recorded as one time, regardless of duration. (Measured in "times during the dtime").

Frequency of nighttime activity (FNA): defined as the number of times a mini-pig shifted from sleep to activity (measured in "times during the nighttime").

Anesthesia: $3 \%$ sodium pentobarbital $(0.5 \mathrm{ml} / \mathrm{kg})$ andxylazine hydrochloride $(0.1 \mathrm{ml} / \mathrm{kg})$ was intramuscularly injected into the left and right buttocks of the mini-pigs. The first administration produced 1 hour to 1.5 hours of anesthesia, therefore xylazine hydrochloride was added as a supplement of $1 / 2$ initial dose.

Locating stomach meridian by low electrical impedance method: After the mini-pigs were anesthetized, the stomach meridian of low electrical impedance was firstly measured by a low impedance meridian locator (type: WQ6F30, made in Donghua Co. in China) with $5 \mu$ s to $10 \mu$ s narrow pulse, $40 \mathrm{~Hz}$ to $80 \mathrm{~Hz}$ and adjustable magnitude. A reference electrode was set on one ear of the mini-pig with a saline cotton ball in the middle. The pen-like testing electrode was moved on the skin perpendicularly to stomach meridian and the electrical current was read on the micro-ampere meter of WQ6F30 instrument. We searched for sites with large electrical currents $(10 \mu \mathrm{A}$ to $50 \mu \mathrm{A}$ ). If none were found after 1 to 2 sweeps, the magnitude could be increased. When a large current was found, it was marked in red. Each time, 15 to 20 points of low electrical impedance were found about $1-2 \mathrm{~cm}$ apart along the stomach meridian, revealing a smooth curved line that represents the stomach meridian of low electrical impedance (Figure 4A).

Locating low hydraulic resistance points: HR was measured using a continuous HR measuring instrument with pressure differential type. At a point at the same height as the point to be measured, we first made a standard wave with HR of zero (opening the valve) and HR at infinitely great (closing the valve). After this, we chose four levers to measure the HR, below the knee joint (near extra point "Housanli (ST36)"), above the knee joint, inguinal groove, and between the first and second nipples counted from above down. The measuring needle was inserted perpendicularly to the low electric impedance line into the loose connective tissue layer below the skin approximately $1.5 \mathrm{~cm}$ apart from the stomach meridian of low electrical impedance line.
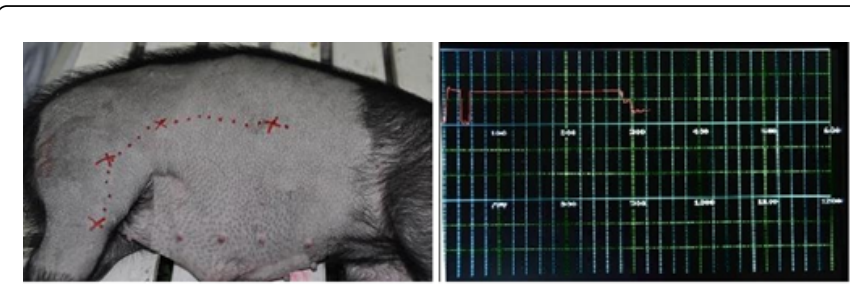

Figure 4: The location of low impedance points(LIP) and low hydraulic resistance points(LHRPs) on the mini-pig. (A) LIP was shown by red round points and LHRP was shown by red $\times$. (B) The hydraulic resistance curve on the screen and a LHRP was shown by 仓.

The needle was slowly and evenly moved forward while monitoring the changes in HR curve on the computer screen. When the needle is moved to a certain site, the hydraulic resistance curve will drop suddenly. At that point, we stop moving the needle, and if the curve remains in the low position then that site is designated as the lower hydraulic resistance point(LHRP, Figure 4B).

Blocking LHRC along the stomach meridian: After finding a LHRP, theneedle was fixed on the skin using AnGuTe quick glue and was separated from the HR measuring system. In the experimental group, $0.5 \mathrm{ml} \sim 1 \mathrm{ml}$ hydro gel was slowly injected into the LHRP which are totally 8 LHRPs on the right and left sides of each animal. Sites below and above the knee joint were injected with $0.5 \mathrm{ml}$ of gel. The point near the inguinal groove was injected with $0.7 \mathrm{ml}$ and the point between the first and second nipples were injected with $1.0 \mathrm{ml}$. On the same sites in the control group, equal amounts of physiological saline were injected into the sites of LHRP. The entire procedure of injection took approximately $30 \mathrm{~s}$ to $1 \mathrm{~min}$. All sites of injection were photographed. After the needles were removed, the pigs were returned to their cages and their behaviors were observed using a 24 hour video surveillance.

Data management: After each bitch of experiment concluded, the video was preserved on the hard drive for analysis. The original video data was played at $\mathrm{x} 32$ speed on a Hanbang video monitor. When an expectant behavior was seen, the video was played at original speed or $\mathrm{x} 1$ speed for a closed observation. Due to the effects of anesthesia, we did not consider the data from the $d$ of experimentation and the $d$ after in order to get stable and accurate results.

Statistical protocols: All statistical data from the experiment was analyzed using SPSS13.0 statistical software. Data was expressed as means \pm standard deviation. Independent-sample $\mathrm{T}$ test was performed on all the data markers between the experimental group and control group. The significant level was $\mathrm{P}<0.05$. Correlation analysis was performed using Pearson bivariate correlation analysis with two-tailed and significant level was $\mathrm{P}<0.05$. For single pig statistics, N-of-1 trials statistical method [7] was used and significant level was set to $\mathrm{P}<0.05$.

\section{Results}

Thirteen mini-pigs were carried in the experiment. They were divided into 4 bitches with total 19752 observing hours. The experimental group included 8 mini-pigs and 5 mini-pigs in control group. FCFD and FNA were collected before modeling $(7 \mathrm{ds})$ and 3 to $41 \mathrm{ds}$ after modeling and then calculated.

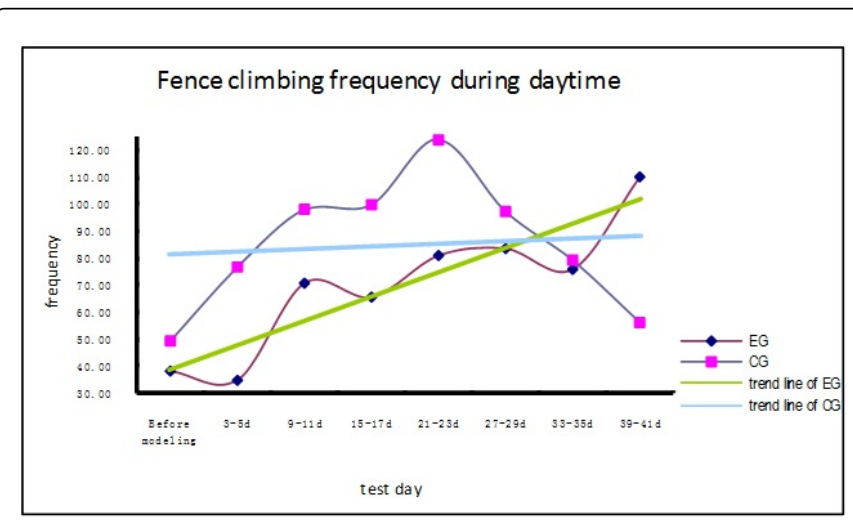

Figure 5: The tendency of FCFD following the ds of the modeling. 
Citation: Zhang WB, Zhou WT, Wang GJ, Jia SY, Tian YY, et al. (2017) Observing Behavioral Effects in Mini-Pigs after Blocking the Low Hydraulic Resistance Channels along the Stomach Meridian. Altern Integr Med 6: 251. doi:10.4172/2327-5162.1000251

Page 4 of 5

Frequency of climbing fence in the dtime

\begin{tabular}{|l|l|l|l|l|l|l|l|l|}
\hline & Before modeling & $3-5$ day & $9-11$ day & $15-17$ day & $21-23$ day & $27-29$ day & $33-35$ day & $39-41$ day \\
\hline Experiment & $38.19 \pm 11.87$ & $34.94 \pm 12.99$ & $70.77 \pm 8.00$ & $65.38 \pm 5.74$ & $81.00 \pm 10.25$ & $83.79 \pm 11.14$ & $75.88 \pm 3.85$ & $109.82 \pm 17.94$ \\
\hline Control & $49.78 \pm 15.91$ & $57.28 \pm 18.59$ & $76.48 \pm 1.61$ & $87.33 \pm 5.57$ & $99.76 \pm 2.31$ & $81.62 \pm 3.73$ & $68.10 \pm 11.15$ & $61.87 \pm 11.84$ \\
\hline
\end{tabular}

Table 1: Frequency of climbing fence in the dtime before and after the modeling.

FCFD before the modeling were averaged during $7 \mathrm{ds}$. FCFD after modeling were averaged in each three ds with three ds interval so that seven averages FCFD were gotten after the modeling. The result was shown in Figure 5 and Table 1.

When comparing the average values one by one, there was no significant difference between the two groups. However, over time, the model group exhibited an upward trend of FCFD which had a correlation coefficient of 0.868 with the modeling ds, i.e. the ds elapsed after establishing the model which was statistically significant $(\mathrm{P}<0.05)$. The control group, by contrast, was -0.045 , which was not statistically significant $(\mathrm{P}>0.05)$.

FNA was calculated similar to FCFD and the result was shown in Table 2 and Figure 6.

Frequency of nighttime activity $(\bar{x}$ Mean $\pm S D)$

\begin{tabular}{|l|l|l|l|l|l|l|l|l|}
\hline & Before modeling & $3-5$ day & $9-11$ day & $15-17$ day & $21-23$ day & $27-29$ day & $33-35$ day & $39-41$ day \\
\hline Experiment & $6.7 \pm 1.23$ & $5.8 \pm 1.93$ & $7.40 \pm 1.82$ & $8.04 \pm 0.69$ & $7.63 \pm 2.34$ & $8.08 \pm 0.95$ & $7.38 \pm 1.52$ & $8.19 \pm 2.21$ \\
\hline Control & $6.9 \pm 0.51$ & $7.8 \pm 0.92$ & $7.71 \pm 1.21$ & $7.57 \pm 0.38$ & $6.90 \pm 0.58$ & $6.76 \pm 0.54$ & $7.48 \pm 0.22$ & $9.67 \pm 1.81$ \\
\hline
\end{tabular}

Table 2: Frequency of nighttime activity before modeling and after modeling.

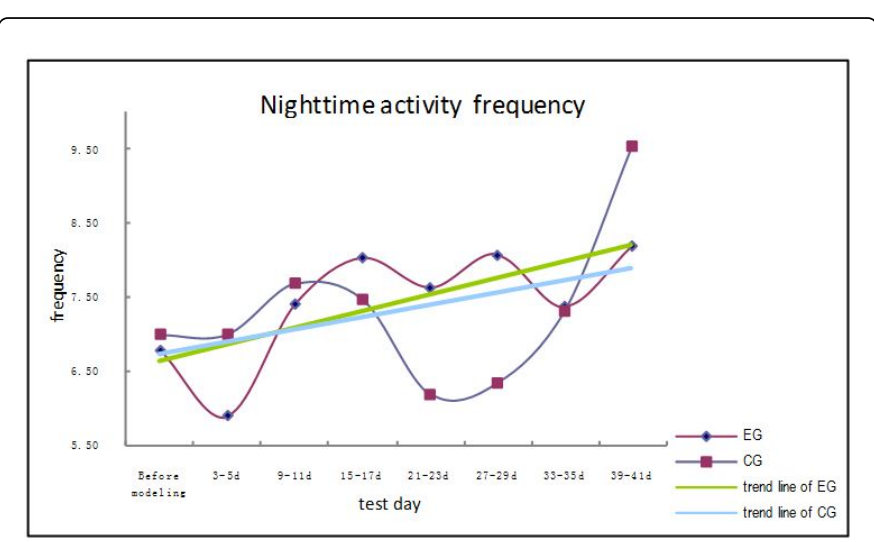

Figure 6: The tendency of FNA following the ds of the modeling.

There was no significant difference on average FNA at one by one between the two groups while the model group exhibited and upward trend of FNA which had a correlation coefficient of 0.675 which is higher compared with a correlation coefficient of 0.401 in control group.

One pair of pigs with a long observation: As the difference of FNA among the experimental mini-pigs was relatively large particularly on behavior, it is difficult to get the statistical result when the number of the animal is small and the housing condition are not sufficient to feed more pigs and keep a constant feeding ds for each pigs. So one pair of pigs, one modeling pig and one control pig, was fed under same condition and observed FNA during a long observing ds which was 21 before modeling and $50 \mathrm{ds}$ after the modeling. The result was shown in Table 3.

\begin{tabular}{|l|l|l|}
\hline \multicolumn{3}{|l|}{ Frequency of nighttime activity $(\overline{\mathbf{x}}$ Mean \pm SD $)$} \\
\hline & Before $(21$ days $)$ & After $(50$ days $)$ \\
\hline Experiment & $5.29 \pm 2.37$ & $8.64 \pm 4.12^{\star *}$ \\
\hline Control & $6.24 \pm 1.99$ & $7.00 \pm 1.95$ \\
\end{tabular}

Table 3: The observation of one pair of pigs during a long observing time.

\section{Discussion}

From above results, it appeared that the frequencies of climbing fence and night activity after modeling were increased following the feeding ds among which FCFD had more obvious correlativity which is 0.868 compared with a negative correlativity $(-0.045)$ in control group. In a single pair of pigs, night activity increased by ds after the modeling while no such tendency on the control pig.

Most studies on meridian and meridian-viscera correlativity concern on physiological or biochemical changes which are easy to be quantitatively measured using an instrument. But the obvious disadvantage is that these indexes are only partial changes in the body. Behavior is the sum of all physiological and biochemical changes in our body. It is also the main observation by ancient Chinese doctor to get the law of disease and therapy. How to measure the behavior particularly on experimental animal is the task of modern research.

Behavior study of animals is getting popular recently. But most studies were carried on mouse and other small animals. Large animals are usually expansive and difficult to be observed so that rarely used in experiments. But sometimes, small animals are difficult to be studied on something, for example to compare the effect of acupuncture on 
Citation: Zhang WB, Zhou WT, Wang GJ, Jia SY, Tian YY, et al. (2017) Observing Behavioral Effects in Mini-Pigs after Blocking the Low Hydraulic Resistance Channels along the Stomach Meridian. Altern Integr Med 6: 251. doi:10.4172/2327-5162.1000251

Page 5 of 5

different body surface. Sometimes, large animals are convenient and a necessary condition to get good results [7]. LHRCM has been found on min-pigs and is hard to be measured on mouse, rat and other small animals whose skin are hairy and soft. So the behavior of pig is the key marker for meridian study.

After observing the behavior of pigs, we found that pigs spend one third time on sleeping or lying and spend other time on climbing fence, pushing by mouth and walking etc. The duration of climbing fence is long and laxative usually. But when a feeder came in, the pig is often getting excited by jumping, climbing fence quickly and cry which might be the behavior of food desire because of a limitation of food amount so as to control the weight. So eating process, including time and amount and climbing behavior is not suitable to be the marker of behavior and frequency of climbing fence in the dtime without feeding and eating was applied as the behavior marker. Through FCFD, the constant spiritual state can be estimated partially. The result showed a gradually increasing of FCFD by time in meridian stasis group which indicate an increasing dysphoric caused by continuous blockage of stomach channel. The phenomenon corresponds well with the description of stomach symptom that when the meridian get a serious sick, the person will climb up a high place and sing or take off cloth and walk.

FNA is a marker of night activity which relates with the quality of sleep. In pathology of TCM, stomach disorder causes a bad sleep. After studying the video of one pair of pigs at night, the pig with stomach stasis appeared many times of awake from sleep indicating a bad sleeping quality, implying a tight relationship between stomach and sleep. In our previous study, the pigs of blocking stomach meridian appear distension of stomach and intestine [5] which might be a reason to disturb the sleep.

The Macrolane gel used in our experiment is a bright hyaluronic acid which has little side effect. Clinical report showed that it may cause a little local tension and sore but release in a short time which did not corresponds to the increasing FCFD and FNA by time. So the changes wouldn't be the stimulation from the injection of gel. Further work could be done on comparing the effects of blocking different meridians.

\section{Conclusion}

Blocking LHRC along stomach meridian produces certain behavioral changes that are consistent with the pathological symptoms of stomach meridian in the classical medical literature on acupuncture. These changes are directly correlated with the modeling ds and gradually become more pronounced with the passage of time.

\section{Acknowledgments}

This work was supported by a grant (No. 81173206) from National Natural Science Foundation of China.

\section{References}

1. Wang B (1997) Yellow Emperor's Canon of Internal Medicine: Ling Su, Jing Mai," Beijing, China Science \& Technology Press, pp: 559.

2. Zhang WB, Tian YY, Li H, Luo MF, Xu FL, et al. (2008) A discovery of low hydraulic resistance channel along meridians. J Acupunct Meridian Stud 1: $20-28$.

3. Xu YH, Zhang WB, Tian YY, Wang LL, Huang T, et al. (2009) Preliminary observation of the blocking effect produced by injecting polyacnylamide hydrogel on low hydraulic resistance channel along meridian. J Biomed Eng 26: 776-779.

4. Zhang WB, Xu YH, Tian YY, Li H, Wang GJ, et al. (2013) Induction of hyperalgesia in pigs through blocking low hydraulic resistance Channels and reduction of the resistance through acupuncture: A mechanism of action of acupuncture. Evid Based Complement Alternat Med.

5. Zhou WT, Jia SY, Zhang YQ, Tian YY, Wang GJ, et al. (2013) Pathological changes in internal organs after blocking low hydraulic resistance channels along the stomach meridian in pigs. Evid Based Complement Alternat Med.

6. Wegman ACM, van der Windt Danielle AWM, Stalman WAB de Vries TPGM (2006) Conducting research in individual patients: lessons learnt from two series of N-of-1 trials. BMC Fam Pract 16: 54.

7. Zhang YQ, Tian YY, Ding YL, Huang T, Jia SY, et al. (2011) An approach to the methods of observing behavioral indexes of experimental minipigs in basic research on traditional Chinese medicine. Lab AnimSci 28: 47-51. 